\title{
A NOTE ON CHARACTERISTIC EQUATION OF TOEPLITZ OPERATORS ON THE SPACES $A_{k}$
}

\author{
GUANGFU CAO
}

\section{Preliminaries}

Let $k$ be any integer, $k \geq 0$. The $k$-th Bergman measure on unit ball $B$ of $C^{n}$, $\mu_{k}$, is given by

$$
d \mu_{k}=\frac{\Gamma(n+k+1)}{\Gamma(n+1) \Gamma(k+1)}\left(1-|w|^{2}\right)^{k} d v(w)
$$

Note that $\mu_{0}$ is simply normalized Lebesque measure on $B$. The $k$-th Bergman space, $A_{k}$, is defined as the space of analytic functions on $B$ which are square integrable with respect to the measure $\mu_{k}$. Note that $A_{k}=H^{2}\left(\mu_{k}\right)$, where $H^{2}\left(\mu_{k}\right)$ be the $L^{2}\left(\mu_{k}\right)$-closure of the ball algebra $A$, and that $A_{j} \subset A_{k}$ for $j \leq k$. The standard orthonormal base for $A_{k}$ is given by

$$
e_{\alpha}^{k}=c(a, k, n) z^{\alpha}=c(a, k, n) r_{1}^{\alpha_{1}} e^{i \alpha_{1} \theta_{1}} \cdots r_{n}^{\alpha_{n}} e^{i \alpha_{n} \theta_{n}}
$$

where $c(\alpha, k, n)$ is a constant number such that $c(\alpha, k, n)\left\|z^{\alpha}\right\|=1$. Let $P_{k}$ denote the projection of $L^{2}\left(\mu_{k}\right)$ onto $A_{k}$. Note that $L^{\infty}\left(\mu_{k}\right)=L^{\infty}(B)=\{f: f$ is essentially bounded on $B$ with respect to Lebesque measure on $B$ \}. Also $H^{\infty}\left(\mu_{k}\right)$, the weak ${ }^{*}$-closure of the polynomials in $z$ in $L^{\infty}(B)$, is the set $\left\{f: f \in L^{\infty}(B)\right.$ and $\left.f A_{k} \subseteq A_{k}\right\}=H^{\infty}$, the set of bounded analytic functions on $B$. For $f \in L^{\infty}(B)$, $\|f\|_{\infty}$ denotes the essential supremum of $f$ on $B$. For any $\varphi \in L^{\infty}(B)$ and for any $k \geq 0$, we define a Toeplitz operator $T_{\varphi}^{(k)}: A_{k} \rightarrow A_{k}$ as follows:

$$
T_{\varphi}^{(k)} f=P_{k}(\varphi f) \quad\left(f \in A_{k}\right) .
$$

It can be seen easily that

$$
T_{\varphi}^{(k)} f(z)=\int_{B} \frac{\varphi(\zeta) f(\zeta)}{(1-\langle z, w\rangle)^{k+n+1}} d \mu_{k}(\zeta)
$$

Received November 8, 1994. 
(consult Rudin [1]). The set of all bounded linear operator on $A_{k}$ is written as $L\left(A_{k}\right)$, clearly, $T_{\varphi}^{(k)} \in L\left(A_{k}\right)$. It is well-known that equation $T_{\bar{z}} T T_{z}=T$ characterize the Toeplitz operators on Hardy space in one complex variable. A. M. Davie and N. P. Jewell [2] proved that $\sum_{i=1}^{n} T_{\bar{z}_{i}} T T_{z_{i}}=T$ characterizes Toeplitz operators on Hardy space of several complex variables. D. H. Yu and Sh. H. Sun [3] proved that $T \in L\left(H^{2}\right)$ is a Toeplitz operator iff equation $T_{\eta}^{*} T T_{\eta}=T$ is hold for each inner function $\eta$. In [4], for $n=1$, N. P. Jewell raised the following.

PROBLEm. Is there a set of operator equations which characterize Toeplitz operators on the weighted Bergman spaces of one complex variable?

In next section, we answer negatively the problem.

\section{Theorems}

THEOREM 1. Let $B$ be a set of operator equations and $A$ be the set of bounded linear operators on the $k$-th weighted Bergman space $A_{k}$ which satisfy $B$. If $A$ contains all Toeplitz operators on $A_{k}$, then $A$ is weak ${ }^{*}$-dense in $L\left(A_{k}\right)$.

Proof. If $A$ is not weak ${ }^{*}$-dense in $L\left(A_{k}\right)$, there exists a nonzero trace class operator $S$ such that $\operatorname{tr}(S T)=0$ for any $T \in A$. Then there exist $\left\{f_{t}\right\}$ in $A_{k}$ such that $S=\sum_{t=1}^{\infty} f_{t} \otimes e_{t}$, where $\left\{e_{t}\right\}$ is the orthonormal basis of $A_{k}$ and $f_{t} \otimes e_{t}$ is a 1 -rank operator on $A_{k}$. Without loss of generality, one can assume that $\left\{e_{t}\right\}_{t=1}^{\infty}=$ $\left\{e_{\alpha}^{k}\right\}_{\alpha \in Z^{+n}}$, where $e_{\alpha}^{k}=c(n, k, \alpha) z^{\alpha}$. For convenience, we replace $f_{i}$ by $f_{\alpha}$. Note $S^{*}=\sum_{\alpha} e_{\alpha}^{k} \otimes f_{\alpha}$, so

$$
S^{*} S=\left(\sum_{\alpha} e_{\alpha}^{k} \otimes f_{\alpha}\right)\left(\sum_{\alpha} f_{\alpha} \otimes e_{\alpha}^{k}\right)=\sum_{\alpha}\left\|f_{\alpha}\right\|_{2}^{2} e_{\alpha} \otimes e_{\alpha}^{k} .
$$

Furthermore, $\|S\|_{C_{1}}=\operatorname{tr}\left(\left(S^{*} S\right)^{\frac{1}{2}}\right)=\Sigma_{\alpha}\left\|f_{\alpha}\right\|_{2}$. Hence, $\Sigma_{\alpha}\left\|f_{\alpha}\right\|_{2} \leq \infty$, consequently, $\sum_{\alpha} f_{\alpha} e_{\alpha}^{k} \in L^{1}$. If $A$ contains all Toeplitz operators on $A_{k}$, then for any $\varphi \in L^{\infty}(B)$, we have $T_{\varphi}^{(k)} \in A$. Thus

$$
\begin{aligned}
\operatorname{tr}\left(T_{\varphi}^{(k)} S\right) & =\sum_{\alpha \in Z^{+n}}\left\langle T_{\varphi}^{(k)} S e_{\alpha}^{k}, e_{\alpha}^{k}\right\rangle \\
& =\sum_{\alpha \in Z^{+n}}\left\langle\varphi\left(\sum_{\beta \in Z^{+n}} f_{\beta} \otimes e_{\beta}^{k}\right) e_{\alpha}^{k}, e_{\alpha}^{k}\right\rangle \\
& =\sum_{\alpha \in Z^{+n}}\left\langle\varphi f_{\alpha}, e_{\alpha}^{k}\right\rangle \\
& =\sum_{\alpha \in Z^{+n}} \int_{B} \varphi f_{\alpha} e_{\alpha}^{\bar{k}} d \mu_{k}
\end{aligned}
$$




$$
=\int_{B} \varphi\left(\sum_{\alpha \in Z^{+n}} f_{\alpha} e_{a}^{\bar{k}}\right) d \mu_{k}=0
$$

Since $\varphi$ is arbitrary, we easily see that $\sum f_{\alpha}(z) e_{a}^{\bar{k}}(z)=0$ for any $z \in B$. Suppose $f_{\alpha}$ has series expansion $f_{\alpha}=\sum_{\beta \in Z^{+n}} a_{\alpha \beta} e_{\beta}^{k}$, then

$$
\begin{aligned}
\sum_{\alpha} f_{\alpha}(z) e_{a}^{\bar{k}}(z) & =\sum_{\alpha} \sum_{\beta} a_{\alpha \beta} e_{\alpha}^{\bar{k}} e_{\beta}^{k}(z) \\
& =\sum_{\alpha}, \sum_{\beta} a_{\alpha \beta} c(n, \alpha, k) c(n, \beta, k) z^{\bar{\alpha}} z^{\beta} \\
& =\sum_{\alpha \beta} a_{\alpha \beta} c(n, \alpha, k) c(n, \beta, k) r^{\alpha+\beta} e^{i(\beta-\alpha) \theta} \\
& =\sum_{t \in Z^{+n}}\left[\sum_{\alpha+\beta=t} a_{\alpha \beta} c(n, \alpha, k) c(n, \beta, k) e^{i(\beta-\alpha) \theta}\right] r^{t}=0,
\end{aligned}
$$

where

$$
\begin{gathered}
\theta=\left(\theta_{1}, \ldots, \theta_{n}\right), 0 \leq \theta_{i} \leq 2 \pi,(\beta-\alpha) \theta=\sum\left(\beta_{i}-\alpha_{\imath}\right) \theta_{\imath}, \\
r=\left(r_{1}, \cdots, r_{n}\right), 0 \leq\|r\|<1 .
\end{gathered}
$$

So for each $t \in Z^{+n}$,

$$
\sum_{\alpha+\beta=t} a_{\alpha \beta} c(n, \alpha, k) c(n, \beta, k) e^{\imath(\beta-\alpha) \theta}=0
$$

i.e.

$$
\sum_{\alpha+\beta=t} a_{\alpha \beta} c(n, \alpha, k) c(n, \beta, k) e^{i(t-2 \alpha) \theta}=0 .
$$

Clearly, $\left\{e^{i(t-2 \alpha) \theta}\right\}$ is linear independent, so $a_{\alpha \beta}=0$ for $\alpha+\beta=t$. Hence, for any $\alpha \in Z^{+n}, \beta \in Z^{+n}$, we have $a_{\alpha \beta}=0$ and so $S=0$. It contradicts that $S \neq 0$. This completes the proof.

Frankfurt [5] proved that no bounded operator $T$ on $A_{0}$ satisfies the operator equation $B_{0}^{*} T B_{0}=T$, where $B_{0}$ is the Bergman shift on $A_{0}(D)$ and $D$ is the unit disc. We can extend this result to the case $A_{k}(B)$. In fact, we have the following.

THEOREM 2. There isn't nonzero bounded operator $T$ on $A_{k}(B)$ such that $\sum_{i=1}^{n} T_{\bar{z}_{i}}^{(k)} T T_{Z_{i}}^{(k)}=T$.

To prove Theorem 2, we need some lemmas. The proof of Lemma 1 is related to that of Proposition 2.4 in [4]. 
Lemma 1. Let $M_{z_{1}} \cdots M_{z_{n}}$ be multiplication by the coordinate functions on $L^{2}\left(B, d \mu_{k}\right)$. If there exists $T \in L\left(L^{2}\right)$ such that $\sum_{i=1}^{n} M_{z_{i}}^{*} T M_{z_{i}}=T$, then $T$ commutes with $M_{z_{i}}, M_{z_{i}}^{*}(i=1, \ldots, n)$.

Proof. For any positive integer $m$ and $f, g \in L^{2}$, we have

$$
\langle T f, g\rangle=\sum_{\Sigma_{i=1}^{n} k_{i}=m} \frac{m !}{k_{1} ! \cdots k_{n} !}\left\langle T M_{z_{1}}^{k_{1}} \cdots M_{z_{n}}^{k_{n}} f, M_{z_{1}}^{k_{1}} \cdots M_{z_{n}}^{k_{n}} g\right\rangle
$$

by $\sum_{i=1}^{n} M_{\bar{z}_{i}} T M_{z_{i}}=T$. Hence

$$
\begin{aligned}
& \left\langle\left(T M_{z_{1}}-M_{z_{1}} T\right) f, g\right\rangle \\
& =\sum_{\Sigma_{i=1}^{n} k_{i}=m} \frac{m !}{k_{1} ! \cdots k_{n} !}\left\langle T M_{z_{1}}^{k_{1}+1} \cdots M_{z_{n}}^{k_{n}} f, M_{z_{1}}^{k_{1}} \cdots M_{z_{n}}^{k_{n}} g\right\rangle \\
& -\sum_{\sum_{i=1}^{n} k_{i}=m} \frac{(m+1) !}{\left(k_{1}+1\right) ! k_{2} ! \cdots k_{n} !}\left\langle T M_{z_{1}}^{k_{1}+1} \cdots M_{z_{n}}^{k_{n}} f, M_{z_{1}}^{*} M_{z_{1}}^{k_{1}+1} \cdots M_{z_{n}}^{k_{n}} g\right\rangle \\
& -\sum_{\sum_{i=2}^{n} k_{i}=m+1} \frac{(m+1) !}{k_{2} ! \cdots k_{n} !}\left\langle T M_{z_{2}}^{k_{2}} \cdots M_{z_{n}}^{k_{n}} f, M_{z_{1}}^{*} M_{z_{2}}^{k_{2}} \cdots M_{z_{n}}^{k_{n}} g\right\rangle \\
& =\sum_{\Sigma_{i=1}^{n} k_{i}=m} \frac{m !}{k_{1} ! \cdots k_{n} !}\left\langle T M_{z_{1}}^{k_{1}+1} \cdots M_{z_{n}}^{k_{n}} f,\left(1-\frac{m+1}{k_{1}+1} M_{z_{1}}^{*} M_{z_{1}}\right) M_{z_{1}}^{k_{1}} \cdots M_{z_{n}}^{k_{n}} g\right\rangle \\
& -\sum_{\Sigma_{i=2}^{n} k_{i}=m+1} \frac{(m+1) !}{k_{2} ! \cdots k_{n} !}\left\langle T M_{z_{2}}^{k_{2}} \cdots M_{z_{n}}^{k_{n}} f, M_{z_{1}}^{*} M_{z_{2}}^{k_{2}} \cdots M_{z_{n}}^{k_{n}} g\right\rangle .
\end{aligned}
$$

Furthermore

$$
\begin{gathered}
\|T\|^{-1}\left\langle\left(T M_{z_{1}}-M_{z_{1}} T\right) f, g\right\rangle \\
\leq \sum_{\Sigma_{i=1}^{n} k_{i}=m} \frac{m !}{k_{1} ! \cdots k_{n} !}\left\|M_{z_{1}}^{k_{1}+1} \cdots M_{z_{n}}^{k_{n}} f\right\| \|\left(1-\frac{m+1}{k_{1}+1} M_{\bar{z}_{1}} M_{z_{1}}\right) M_{z_{1}}^{k_{1}} \cdots M_{z_{n}}^{k_{n} g \|} \\
+\sum_{\Sigma_{i=2}^{n} k_{i}=m+1} \frac{(m+1) !}{k_{2} ! \cdots k_{n} !}\left\|M_{z_{2}}^{k_{2}} \cdots M_{z_{n}}^{k_{n}} f,\right\|\left\|M_{\bar{z}_{1}} M_{z_{2}}^{k_{2}} \cdots M_{z_{n}}^{k_{n}} g\right\| .
\end{gathered}
$$

Note for any $f \in L^{2}\left(B, d \mu_{k}\right)$

$$
\begin{aligned}
& \left\|\left(M_{\bar{z}_{1}} M_{z_{1}}+\cdots+M_{\bar{z}_{n}} M_{z_{n}}\right)^{m} f\right\| \\
= & \left\|\left(\sum_{\Sigma_{i=1}^{n}\left|z_{i}\right|^{2}}\right)^{m} f\right\| \rightarrow 0 \quad(m \rightarrow \infty)
\end{aligned}
$$

and

$$
\sum_{\Sigma_{i=1}^{n} p_{i}=m} \frac{m !}{p_{1} ! \cdots p_{n} !}\left\|M_{z_{1}}^{p_{1}} \cdots M_{z_{n}}^{p_{n}} f\right\|^{2}
$$




$$
\begin{aligned}
& =\sum_{\Sigma_{i=1}^{n} p_{i}=m} \frac{m !}{p_{1} ! \cdots p_{n} !}\left\langle\left(M_{\bar{z}_{1}} M_{z_{1}}\right)^{p_{1}} \cdots\left(M_{\bar{z}_{n}} M_{z_{n}}\right)^{p_{n}} f, f\right\rangle \\
& =\left\langle\left(M_{\bar{z}_{1}} M_{z_{1}}+\cdots+M_{\bar{z}_{n}} M_{z_{n}}\right)^{m} f, f\right\rangle \\
& \leq\left\|\left(\sum_{\Sigma_{i=1}^{n}\left|z_{i}\right|^{2}}\right)^{m} f\right\|\|f\| .
\end{aligned}
$$

By

$$
\begin{gathered}
\sum_{\Sigma_{i=1}^{n} k_{i}=m} \frac{m !}{k_{1} ! \cdots k_{n} !}\left\|M_{z_{1}}^{k_{1}+1} \cdots M_{z_{n}}^{k_{n}} f\right\|\left\|\left(1-\frac{m+1}{k_{1}+1} M_{z_{1}} M_{z_{1}}\right) M_{z_{1}}^{k_{1}} \cdots M_{z_{n}}^{k_{n}} g\right\| \\
\leq\left[\sum_{\Sigma_{i=1}^{n} k_{i}=m} \frac{m !}{k_{1} ! \cdots k_{n} !} \frac{m+1}{k_{1}+1}\left\|M_{z_{1}}^{k_{1}+1} \cdots M_{z_{n}}^{k_{n}} f\right\|^{2}\right]^{\frac{1}{2}} \\
\left.\sum_{\sum_{i=1}^{n} k_{i}=m} \frac{m !}{k_{1} ! \cdots k_{n} !} \frac{k_{1}+1}{m+1}\left\|\left(1-\frac{m+1}{k_{1}+1} M_{z_{1}} M_{z_{1}}\right) M_{z_{1}}^{k_{1}} \cdots M_{z_{n}}^{k_{n}} g\right\|^{2}\right]^{\frac{1}{2}}
\end{gathered}
$$

and

$$
\begin{aligned}
\sum_{\sum_{i=1}^{n} k_{i}=m} & \frac{m !}{k_{1} ! \cdots k_{n} !} \frac{k_{1}+1}{m+1}\left\|\left(1-\frac{m+1}{k_{1}+1} M_{\bar{z}_{1}} M_{z_{1}}\right) M_{z_{1}}^{k_{1}} \cdots M_{z_{n}}^{k_{n}} g\right\|^{2} \\
= & \sum_{\Sigma_{i=1}^{n} k_{i}=m} \frac{m !}{k_{1} ! \cdots k_{n} !} \frac{k_{1}+1}{m+1}\left[\left\|M_{z_{1}}^{k_{1}} \cdots M_{z_{n}}^{k_{n}} g\right\|^{2}\right. \\
& \quad-2 \frac{m+1}{k_{1}+1} R e\left\langle M_{z_{1}}^{k_{1}} \cdots M_{z_{n}}^{k_{n}} g, M_{\bar{z}_{1}} M_{z_{1}} M_{z_{1}}^{k_{1}} \cdots M_{z_{n}}^{k_{n}} g\right\rangle \\
& \left.+\left(\frac{m+1}{k_{1}+1}\right)^{2}\left\|M_{\bar{z}_{1}} M_{z_{1}} M_{z_{1}}^{k_{1}} \cdots M_{z_{n}}^{k_{n}} g\right\|^{2}\right] \\
\leq & \sum_{\sum_{i=1}^{n} k_{i}=m} \frac{m !}{k_{1} ! \cdots k_{n} !}\left[\left\|M_{z_{1}}^{k_{1}} \cdots M_{z_{n}}^{k_{n}} g\right\|^{2}+2\left\|M_{z_{1}}^{k_{1}} \cdots M_{z_{n}}^{k_{n}} g\right\|^{2}\right. \\
& \left.+\frac{m+1}{k_{1}+1}\left\|M_{z_{1}}^{k_{1}+1} M_{z_{2}}^{k_{2}} \cdots M_{z_{n}}^{k_{n}} g\right\|^{2}\right] \\
\leq & \left.3\left\langle\sum_{i=1}^{n} M_{\bar{z}_{i}} M_{z_{i}}\right)^{m} g, g\right\rangle+\left\langle\left(\sum_{i=1}^{n} M_{\bar{z}_{i}} M_{z_{i}}\right)^{m+1} g, g\right\rangle \\
\leq & \left.3\left\|\left(\sum_{i=1}^{n} M_{\bar{z}_{i}} M_{z_{i}}\right)^{m} g\right\| .\|g\|+\| \sum_{i=1}^{n} M_{\bar{z}_{i}} M_{z_{i}}\right)^{m+1} g\|.\| g \|,
\end{aligned}
$$

we have

$$
T M_{z_{1}}-M_{z_{1}} T=0
$$

i.e.

$$
T M_{z_{1}}=M_{z_{1}} T
$$


Similarly, $T M_{z_{i}}=M_{z_{i}} T$, for $i=1,2, \cdots, n$. It shows the lemma.

Lemma 2. If $T \in L\left(A_{k}\right)$ satisfy $\sum_{i=1}^{n} T_{\bar{z}_{i}} T T_{z_{i}}=T$. Then there is $S \in$ $L\left(L^{2}\right)$ with $\|S\|=\|T\|, \sum_{i=1}^{n} M_{\bar{z}_{i}} S M_{z_{i}}=S$ and such that $T$ is the compression of $S$ to $A_{k}$.

Proof. It is similar to the proof of Lemma 2.5 in [2]. In fact, we can define $\phi$ : $L\left(L^{2}\right) \rightarrow L\left(L^{2}\right)$ by

$$
\phi(S)=\sum_{i=1}^{n} M_{\bar{z}_{i}} S M_{z_{i}}
$$

then $\|\phi(S)\| \leq\|S\|$. Let $T^{\sim}$ be any operator on $L^{2}$ whose compression is $T$, with $\left\|T^{\sim}\right\|=\|T\|$, let $S_{m}=\frac{1}{m} \sum_{i=1}^{m} \phi^{i}\left(T^{\sim}\right)$, and let $S$ be a weak operator topology limit point of $\left\{S_{m}\right\}$, then $S$ has the required properties.

Lemma 3. If $T \in L\left(A_{k}\right)$ satisfies $\sum_{i=1}^{n} T \frac{k}{z_{i}} T T_{z_{k}}^{k}=T$, then $T$ is a Toeplitz operator.

Proof. If $T$ satisfies the equation, and $S$ is the operator given by Lemma 2, then Lemma 1 shows that $S$ commutes with $M_{z_{k}}$ and $M_{\bar{z}_{k}}\{k=1, \ldots, n)$, so there is $\varphi \in L^{\infty}$ such that $S=M_{\varphi}$, consequently, $T=T_{\varphi}^{(k)}$.

Proof of Theorem 2. If there is $T \in L\left(A_{k}\right)$ such that $\sum_{i=1}^{n} T_{\bar{z}_{i}}^{k} T T_{z_{i}}^{(k)}=T$, then $T$ is a Toeplitz operator on $A_{k}$, i.e., there is $L^{\infty}$, such that $T=T_{\varphi}^{(k)}$. Note

$$
\sum_{i=1}^{n} T_{\bar{z}_{i}}^{(k)} T_{\varphi}^{(k)} T_{\bar{z}_{i}}^{(k)}=T_{\left(\Sigma_{i=1}^{n}\left|z_{i}\right|^{2}\right) \varphi}^{(k)}
$$

so $T_{\left(\Sigma_{i=1}^{n}\left|z_{i}\right|^{2}\right) \varphi}^{(k)}=T_{\varphi}^{(k)}$, and hence, $T_{\left(1-\Sigma_{i=1}^{n}\left|z_{i}\right|^{2}\right) \varphi}^{(k)}=0$. Hence, $\varphi=0$, consequently, $T=$ 0 . We complete the proof of Theorem 2 .

The author is indebted to the referee for his many suggestions.

\section{REFERENCES}

[1] W. Rudin, Function Theory in the Unit Ball of $C^{n}$, Springer-verlag, New York Heidelberg Berlin, 1980.

[2] A. M. Davie and N. P. Jewell, Toeplitz operators in several complex variables., J. Funct. Anal., 26 (1977), 356-368. 
[ 3 ] D. H. Yu and Sh. S. Sun, The $w^{*}$-closure of operator space, Acta Math. of China (to appear).

[4] N. P. Jewell, Toeplitz operators on the Bergman spaces and in several complex Variables, Proc. London Math. Soc., 41 (1980), 193-216.

[5] R. Frankfurt, Operator equations and weigted shifts, J. Funct. Anal., 62 (1978) $610-619$.

Department of Mathematics

Sichuan University

Chengdu 610064, China 